\title{
IDENTIFICAÇÃO DE EXTRAÍVEIS VOLÁTEIS PRESENTES NA EMBALAGEM PRIMÁRIA DE UM SPRAY NASAL
}

\author{
Ângela Oliveira Francisco ${ }^{1,2^{*}}$, Celso Blatt $^{3}$, Daniele de Souza Freitas ${ }^{2}$, Paulo R. H. Moreno ${ }^{1^{*}}$ \\ 1 - Instituto de Química, Universidade de São Paulo (USP), São Paulo, SP \\ 2 - Ache Laboratórios Farmacêuticos \\ 3 - Agilent Technologies do Brasil \\ prmoreno@iq.usp.br; angela.francisco@ache.com.br
}

\begin{abstract}
Resumo: Extraíveis são substâncias químicas orgânicas e inorgânicas, liberadas de um sistema de embalagem utilizado para acondicionar um medicamento, em condições de laboratório. Essas substâncias têm o potencial de lixiviar para um produto farmacêutico nas condições normais de armazenamento durante seu período de validade, sendo portanto, denominadas de lixiviáveis. Este trabalho investigou a composição química de origem volátil presente nos componentes individuais do material de embalagem de um spray nasal em análise por headspace em cromatógrafo a gás acoplado à espectrometria de massas (CG-EM). A identificação destes compostos foi obtida através da comparação dos índices de retenção linear, dos espectros de massa obtidos com as bibliotecas instaladas no equipamento e da literatura, utilizando uma série homóloga de $n$-alcanos, analisada nas mesmas condições experimentais. Os principais compostos presentes em todos os componentes foram dodecano e tetradecano, seguidos de decano e 2, 2, 4, 6, 6-pentametil-heptano. Estes resultados possibilitam uma correlação com os potenciais lixiviáveis, para estabelecer um protocolo de controle de qualidade para o acompanhamento destas substâncias no medicamento, aumentando sua segurança e eficácia, através da avaliação da sua toxicidade, se presentes no medicamento.
\end{abstract}

Palavras-chave: spray nasal, compostos voláteis, extraíveis, índice de retenção linear.

\section{IDENTIFICATION OF VOLATILE EXTRACTABLES IN THE PRIMARY PACKAGING OF A NASAL SPRAY}

\begin{abstract}
Extractables are organic and inorganic chemical substances, released from a packaging system, under laboratory conditions. These substances have potential to leach into a pharmaceutical product under normal storage conditions during its shelf life, being called by leachables. This work investigated the chemical composition of volatile origin present in the individual components of the packaging material of a nasal spray, which were packaged in a vial and analyzed by headspace in a gas chromatograph coupled to mass spectrometry (GC-MS). The identification of these compounds was obtained by a comparison between linear retention index, the mass spectra obtained with the libraries installed in the equipment (NIST 08/Willey 2014) and literature, using a homologous series of $b$-alkanes (Sigma Aldrich, C8 -C40), analyzed under the same experimental conditions. The main compounds present in all components were dodecane and tetradecane, followed by decane and heptane, 2, 2, 4, 6, 6-pentamethyl. These results enable a correlation with the potential leachables, and to establish a quality control protocol for monitoring these substances in the drug, increasing its safety and efficacy, through the assessment of their toxicity, if present in the drug.
\end{abstract}

Keywords: nasal spray, volatile compounds, extractables, linear retention index.

\section{Introdução}

O sistema de embalagem deve ser adequado para o seu objetivo de uso, ou seja, deve proteger adequadamente a forma farmacêutica; ser compatível com a dosagem; ter em sua composição materiais considerados seguros para uso conforme a dosagem e a via de administração. Além disso, muitos desses dispositivos são projetados para liberar adequadamente o número de doses, e portanto, precisam demonstrar conformidade de desempenho. ${ }^{1}$ 
Uma variedade de materiais, como vidro, metais, materiais sintéticos ou polímeros naturais são usados para fabricar componentes de embalagem e dispositivos médicos. Destes, os mais frequentemente utilizados são provavelmente materiais poliméricos, isto é, materiais que consistem em moléculas de cadeia longa e/ou reticulada, compostas por unidades chamadas monômeros. ${ }^{2}$

Uma impureza é qualquer componente do medicamento que não seja a substância medicamentosa ou um excipiente. Durante a produção e armazenamento do medicamento, podem ocorrer impurezas desde o processo de fabricação, devido à falta de estabilidade da substância medicamentosa, ou como resultado de incompatibilidade com excipientes. Impurezas podem ser classificadas nos seguintes grupos: impurezas orgânicas (voláteis, semi-voláteis e não voláteis), impurezas inorgânicas e solventes residuais. ${ }^{2}$ Alguns componentes provenientes do processo de manufatura do material de embalagem podem migrar para o produto farmacêutico, formando uma classe de impurezas conhecidas como lixiviáveis. ${ }^{3}$ Já os extraíveis são substâncias químicas orgânicas e inorgânicas que são liberadas de um sistema de embalagem, em um solvente de extração em condições de laboratório. Essas substâncias têm o potencial de lixiviar para um produto farmacêutico nas condições normais de armazenamento e no seu período de validade, sendo, portanto, denominadas de lixiviáveis. ${ }^{4}$

A via de administração deve ser considerada ao avaliar o risco potencial de um lixiviável, porque sua toxicidade depende da rota de entrada no organismo. Por exemplo, um mesmo composto pode ser oralmente ingerido com segurança em determinados níveis pré-estabelecidos, mas pode ter um efeito tóxico se o mesmo nível for inalado diretamente nos pulmões. As vias de administração de maior preocupação são as vias inalatória, injetável, seguida da oftálmica, transdérmica e nasal. ${ }^{5}$

Com a finalidade de otimizar os resultados são empregados métodos ortogonais para maximizar a probabilidade de que todos os extraíveis predominantes sejam detectados e devidamente avaliados. ${ }^{6}$ A primeira abordagem é avaliar os componentes voláteis presentes em um sistema de embalagem, pois há probabilidade de que esses compostos lixiviem para a formulação. $\mathrm{O}$ método mais usado nessa análise é o headspace em cromatógrafo a gás acoplado à espectrometria de massas (CG-EM).

Dessa maneira, o objetivo deste trabalho é identificar as substâncias voláteis presentes nos materiais poliméricos que compõem o sistema de embalagem primária de uma formulação aquosa de um glicocorticoide de uso nasal. O spray nasal proposto neste estudo, possui um sistema de embalagem primária composto por material polimérico e metálico, sendo frasco de polietileno (PE), válvula de polipropileno (PP), copolímero de etileno com acetato de vinila (EVA) e fios de prata. Com esses resultados teremos os primeiros passos para propor um protocolo de controle de qualidade para o acompanhamento destas substâncias, aumentando sua segurança e eficácia, através da avaliação da toxicidade dos lixiviáveis, se presentes no medicamento.

\section{Experimental}

O sistema de embalagem completo, e a nomenclatura referente a cada componente do material de embalagem analisado, são indicados na Figura 1. 


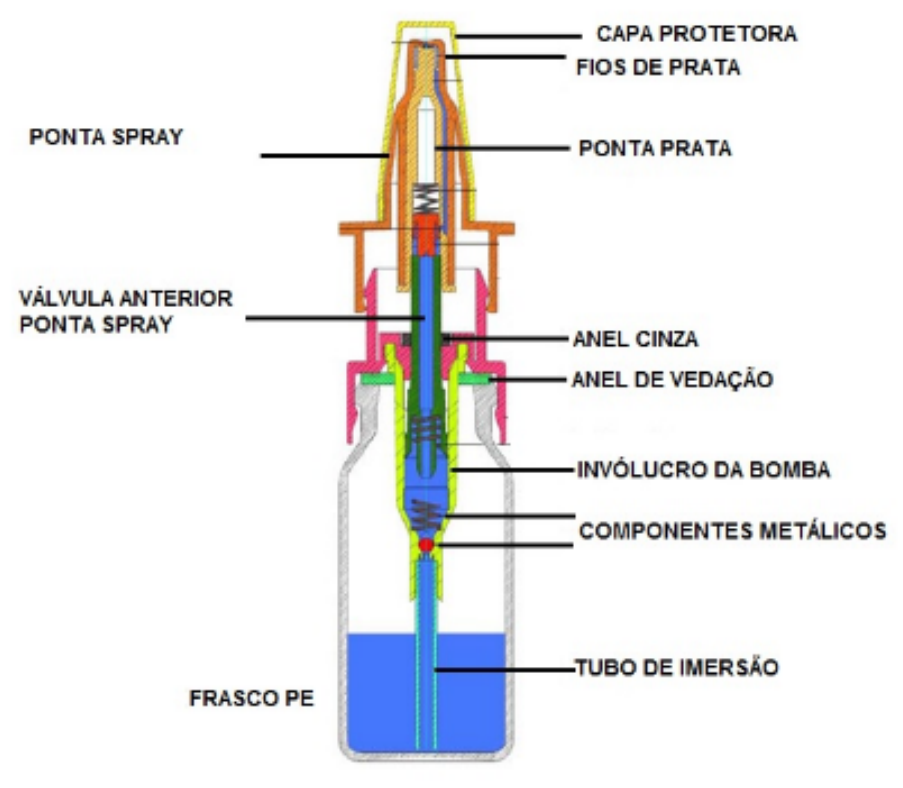

Figura 1 - Sistema de embalagem do spray nasal, adaptado ${ }^{7}$

Os frascos para análise de headspace foram previamente descontaminados por aquecimento em estufa à $300^{\circ} \mathrm{C}$. Os componentes foram então pesados e acondicionados, sendo 1 componente inteiro por frasco, exceto para o frasco de PE, com massas que variam de $0,016 \mathrm{~g}$ até $3,0 \mathrm{~g}$, em triplicata, conforme Figura 2:
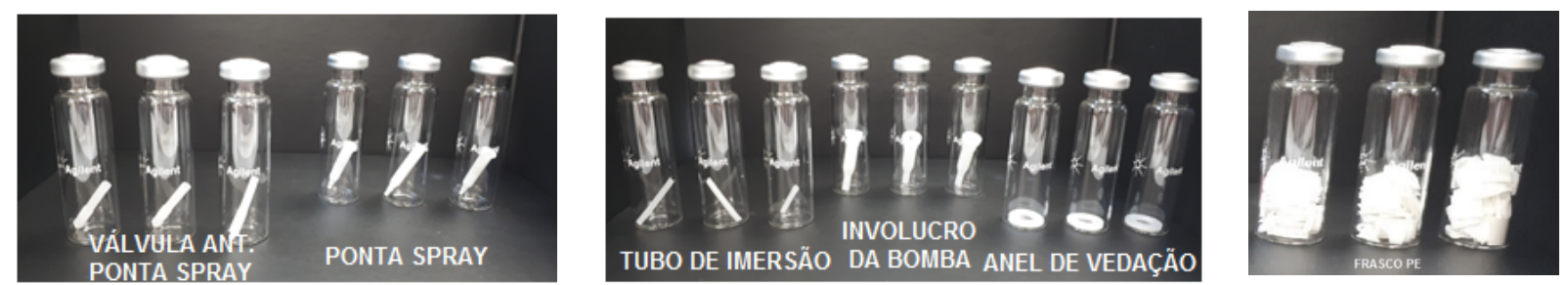

Figura 2 - Acondicionamento dos componentes individuais para análise por headspace em cromatógrafo a gás acoplado à espectrometria de massas

Os componentes foram analisados individualmente por Headspace 7697A em Cromatógrafo a gás Agilent 7890B Series GC (Agilent Santa Clara, CA, USA) acoplado a detector de massas 5977B Quadrupolo (Agilent Santa Clara, CA, USA) e equipado com coluna HP-5MS (30m x 0.25mm x $0.25 \mu \mathrm{m}$ ) (Agilent, Santa Clara, CA, USA). As condições cromatográficas seguiram conforme descrito: $40^{\circ} \mathrm{C}$ por 1 minuto, com aquecimento subsequente de $10^{\circ} \mathrm{C} /$ minuto até $300^{\circ} \mathrm{C}$, manteve-se esta temperatura final por 10 minutos; gás de arraste Hélio fluxo de 1,2 $\mathrm{mL} /$ minuto; temperatura do injetor de $125^{\circ} \mathrm{C}$; modo split $1: 20$, liner $250 \mu \mathrm{L}$, temperatura do forno $120^{\circ} \mathrm{C}$ por 30 minutos, tempo de aquecimento dos vials 30 minutos, temperatura da primeira linha de transferência $130^{\circ} \mathrm{C}$, temperatura da segunda linha de transferência $310^{\circ} \mathrm{C}$, fonte de ionização por elétrons $70 \mathrm{eV}$, 
temperatura da fonte $300^{\circ} \mathrm{C}$, temperatura do quadrupolo $150^{\circ} \mathrm{C}$, intervalo de detecção do MS 33-600 $(\mathrm{m} / \mathrm{z})$.

O índice de retenção de linear (IR) foi calculado de acordo com Dool e Kratz ${ }^{8}$, utilizando uma série homóloga de $n$-alcanos (Sigma Aldrich, C8-C40) submetidas às mesmas condições de análise cromatográfica das amostras. A identificação dos compostos voláteis foi realizada através da comparação dos índices de retenção, espectros de massa obtidos com as bibliotecas instaladas no equipamento (NIST 08/Willey 2014) e literatura ${ }^{9,10}$.

A avaliação de toxicidade para os compostos identificados foi feita através do software de predição in sílico Derek/Sarah ${ }^{\circledR}$ da Lhasa Limited (Leeds, Reino Unido, Nexus 2.3.0).

\section{Resultados e discussão}

A Tabela 1 contém a composição química encontrada para os compostos voláteis presentes no material polimérico, obtidos por componente individual do material de embalagem. Os compostos com menos de 8 carbonos não tiveram seus índices de retenção calculados, pois o primeiro alcano da série de padrões foi o $n$-octano cujo índice de retenção é 800 .

Os compostos dodecano e tetradecano foram obtidos em todos os componentes individuais do sistema de embalagem, seguidos de decano e 2, 2, 4, 6, 6-pentametil-heptano, ausentes apenas no frasco de polietileno. A maioria dos compostos listados na Tabela 1 são hidrocarbonetos alifáticos, provenientes do processo de síntese dos componentes poliméricos.

Tabela 1. Compostos voláteis identificados em CG-EM pela biblioteca de espectros de massa e índice de retenção linear

\begin{tabular}{|c|c|c|c|c|c|c|c|c|c|c|}
\hline \multirow{3}{*}{ Composto } & \multirow{3}{*}{$\mathbf{I R}^{\mathbf{a}}$} & \multicolumn{9}{|c|}{ Parte da Embalagem } \\
\hline & & \multirow[t]{2}{*}{ IR $_{\text {Lit. }}{ }^{b}$} & \multicolumn{7}{|c|}{ \% Área } & \multirow[b]{2}{*}{ PS } \\
\hline & & & PE & TI & IB & AV & $\mathrm{AC}$ & VPS & $\mathbf{P P}$ & \\
\hline 2-metil-1-penteno* & - & 584 & - & - & - & - & - & - & 0,9 & - \\
\hline 1,3,5-trioxano* & - & 650 & - & - & - & - & - & - & - & 6,1 \\
\hline Heptano* & - & 700 & - & - & 2,0 & 2,9 & 0,5 & - & - & - \\
\hline 4-metil-heptano* & - & 764 & - & - & - & - & - & - & 4,6 & - \\
\hline 2,4-dimetil-1-heptano & 822 & 822 & - & - & 17,6 & - & - & - & - & - \\
\hline 2,4-dimetil-1-hepteno & 841 & 842 & - & - & - & - & - & - & 12,0 & - \\
\hline 4-metil-octano & 861 & 864 & - & - & - & - & - & - & 1,7 & - \\
\hline 2, 2, 4, 6, 6-pentametil-heptano & 993 & 995 & - & 9,9 & 6,9 & 6,6 & 12,4 & 3,9 & 3,1 & 7,9 \\
\hline decano & 1001 & 1000 & - & 7,0 & 4,9 & 6,7 & 18,7 & 7,4 & 7,7 & 5,0 \\
\hline NI & 1011 & - & - & - & - & - & - & - & 2,3 & - \\
\hline NI & 1014 & - & - & - & - & - & - & - & 0,2 & - \\
\hline 2,6-dimetil-nonano & 1015 & 1022 & - & - & - & - & - & - & 2,4 & - \\
\hline 2,2,4,4-tetrametiloctano & 1031 & 1045 & - & - & - & - & 2,6 & - & 0,5 & - \\
\hline NI & 1053 & - & - & - & - & - & - & - & 1,0 & - \\
\hline NI & 1060 & - & - & - & - & - & 7,2 & - & 8,0 & - \\
\hline 2-metil-decano & 1066 & 1069 & 1,4 & - & - & - & 5,3 & 0,8 & - & - \\
\hline
\end{tabular}

*: compostos identificados apenas pelo espectro de massas, á́ndice de Retenção Calculado, bÍndice de Retenção da Literatura, PE: frasco de polietileno, TI: tubo de imersão, IB: invólucro da bomba, AV: anel de vedação, AC: anel cinza, VPS: válvula da ponta do spray, PP: ponta próxima ao fio de prata, PS: ponta spray, NI: não identificado

Cont. 
Tabela 1. Cont.

\begin{tabular}{|c|c|c|c|c|c|c|c|c|c|c|}
\hline \multirow{3}{*}{ Composto } & \multirow{3}{*}{$\mathbf{I R}^{\mathbf{a}}$} & \multicolumn{9}{|c|}{ Parte da Embalagem } \\
\hline & & \multirow[t]{2}{*}{ IR $_{\text {Lit. }}{ }^{\mathbf{b}}$} & \multicolumn{7}{|c|}{ \% Área } & \multirow[b]{2}{*}{ PS } \\
\hline & & & $\mathbf{P E}$ & TI & IB & $\mathbf{A V}$ & $\mathbf{A C}$ & VPS & $\mathbf{P P}$ & \\
\hline NI & 1075 & - & - & - & - & - & - & - & 0,3 & - \\
\hline NI & 1082 & - & - & - & - & - & 4,0 & - & 4,7 & - \\
\hline NI & 1097 & - & - & - & - & - & - & - & 2,5 & - \\
\hline undecano & 1101 & 1100 & 4,5 & - & - & - & 5,5 & - & - & - \\
\hline NI & 1104 & - & - & - & - & 3,8 & - & 1,8 & 11,6 & - \\
\hline NI & 1107 & - & - & 2,1 & 1,5 & - & - & - & - & - \\
\hline 4-metil-undecano & 1117 & 1160 & - & - & - & - & 5,1 & - & 4,3 & - \\
\hline 5-2-metilpropil nonano & 1130 & 1185 & - & - & - & - & 0,2 & - & - & - \\
\hline 2-metil-undecano & 1166 & 1164 & - & - & - & - & 0,6 & - & - & - \\
\hline dodecano & 1201 & 1200 & 52,3 & 35,4 & 24,8 & 27,9 & 36,5 & 29,5 & 10,2 & 38,6 \\
\hline 1,3-bis (1,1-dimetil etil) benzeno & 1260 & 1249 & 6,7 & - & - & - & - & - & - & - \\
\hline tridecano & 1302 & 1300 & 3,4 & - & - & - & - & - & - & - \\
\hline NI & 1309 & - & - & - & - & - & - & - & 2,1 & - \\
\hline NI & 1323 & - & - & - & - & - & - & - & 1,0 & - \\
\hline 2,7,10-trimetil-dodecano & 1329 & 1320 & - & - & - & - & - & - & 8,0 & - \\
\hline NI & 1346 & - & - & - & - & - & - & - & 1,9 & - \\
\hline 4,6-dimetil-dodecano & 1348 & 1325 & - & - & - & - & - & - & 1,4 & - \\
\hline tetradecano & 1402 & 1400 & 31,7 & 45,6 & 32,0 & 44,3 & 1,3 & 29,8 & 5,6 & 32,8 \\
\hline 2-bromo-dodecano & 1509 & 1493 & - & - & - & - & - & - & 0,2 & - \\
\hline hexadecano & 1602 & 1600 & - & - & 10,2 & 7,7 & - & 18,1 & 1,8 & 9,6 \\
\hline octadecano & 1803 & 1800 & - & - & - & - & - & 8,7 & - & - \\
\hline
\end{tabular}

*: compostos identificados apenas pelo espectro de massas, ándice de Retenção Calculado, bÍndice de Retenção da Literatura, PE: frasco de polietileno, TI: tubo de imersão, IB: invólucro da bomba, AV: anel de vedação, AC: anel cinza, VPS: válvula da ponta do spray, PP: ponta próxima ao fio de prata, PS: ponta spray, NI: não identificado

Através da predição toxicológica feita pelo software, foi atribuída uma classificação com respeito ao potencial mutagênico e carcinogênico dos compostos, baseada nas diretrizes do ICH $\mathrm{M} 7^{11}$. Nesta avaliação, todos os compostos voláteis foram classificados como classe 5 , exceto 1,3,5-trioxano e 2-bromo-decano, pertencentes à classe 3. Aqueles classificados como classe 5, não possuem alertas estruturais para mutagenicidade. Contudo, os classe 3 poderão ser reclassificados, após avaliação dos dados obtidos por um toxicologista. O controle destas impurezas será necessário, se de fato estes compostos forem lixiviáveis, ou seja, estiverem presentes no medicamento.

\section{Conclusões}

A análise dos compostos voláteis presentes no material de embalagem primária de um spray nasal, identificou a presença majoritária de hidrocarbonetos alifáticos provenientes do processo de síntese do material polimérico. A avaliação de toxicidade dos compostos voláteis encontrados foi obtida através da predição in silico, indicando que maioria dos compostos não possuem alerta estrutural para mutagenicidade (classe 5). Apenas dois compostos apresentaram um alerta de classe 
3 que necessitam revisão de um toxicologista para atribuição de um limite de controle, caso estes compostos sejam posteriormente encontrados como lixiviáveis.

\section{Agradecimentos}

Agradecemos ao apoio do Aché Laboratórios Farmacêuticos, Agilent Technologies do Brasil e Instituo de Química da Universidade de São Paulo.

\section{Referências}

1. https://www.fda.gov/regulatory-information/search-fda-guidancedocuments/containerclosure-systems-packaging-human-drugs-and-biologics, disponível em outubro de 2021.

2. Rodriguez, L., et al. International Journal of Pharmaceutics 2020, 583. https://doi.org/10.1016/j.ijpharm.2020.119332.

3. Jenke, D. et al. PDA J Pharm Sci and Tech 2017, 71, 68.

4. https://online.uspnf.com/uspnf/document/1_GUID-5B829ECA-165E-46C5-A2443FF958BBC190_3 en-US? source $=$ Quick\%20Search\&highlight $=1663$, disponível em outubro de 2021.

5. Moyer, K. L., et al. Specification of Drug Substances and Products : Extractables and Leachables. Oxford : Elsevier 2020. https://doi.org/10.1016/B978-0-08-102824-7.00020-8

6. Jenke, D., et al. PDA Journal Pharmaceutical Science and Technology 2013, 67, 448-511.

7. Klocker, N., et al. European Journal of Pharmaceutics and Biopharmaceutics 2004, 489-493.

8. Dool, V. D. H., Kratz, P. D. Journal Chromatography A 1963, 11, 463-471. https://doi.org/10.1016/S0021-9673(01)80947-X

9. Adams, R. P. Identification of Essential Oil Components by Gas Chromatography Mass Spectrometry. Academic Press: New York 2007. ISBN 978-1-932633-21-4

10. NIST. National Institute of Standards and Technology. US. Department of Commerce. https://webbook.nist.gov, disponível em novembro de 2021.

11. https://www.ema.europa.eu/en/ich-m7-assessment-control-dna-reactive-mutagenicimpurities-pharmaceuticals-limit-potential, disponível em novembro de 2021. 